At first the Thorax editorial office was based at the BTS, before moving to BMA House, and we are grateful to our first Editorial Assistant Ed Howard who guided us so well in changing to a new on line submission system for Thorax and who managed the journal so efficiently for us until 2006. Ed was also very talented in enabling some of the more 'social' aspects of the journal, and we recall some very pleasant dinners both at the annual Thorax editors meetings and during the ATS meeting. We would like to thank Julia Dimitriou who took over from Ed and has so very conscientiously managed the journal for us, providing constant support to the whole editorial team, and we are delighted that she has been recently promoted to the post of Thorax Production Editor. In 2008, David Mitchell announced his resignation from the Thorax editorship after 5 years of very dedicated contribution to the journal. We are grateful to all the technical editors who have contributed to making the journal look so professional each month and especially to Liz Stockman who had been the Thorax technical editor for many years, pre-dating our editorship, till 2007. We would like to thank our Thorax managers, initially Andrea Horgan who provided us with many ideas to enhance the journal and also taught us a great deal about publishing, and lately Claire Folkes who has so expertly managed Thorax for us. We would like to thank Alex Williamson Publishing Manager of BMJ Journals who retired at the end of 2007. A number of Thorax editors have had the immense privilege to work with Alex over the years; she was very fond of the journal and gave us all much valued wise counsel. She was instrumental in developing Thorax into the high quality international journal it is today. We are also grateful to Peter Ashman who is the current Publishing Manager.

The peer reviewers are especially important to a journal, and constructive criticism of a paper greatly improves the final published version. Each year we have published the name of the reviewers who helped us over the preceding year and we would like to express our gratitude to all the peer reviewers for helping us to select the very best papers for publication. Unfortunately over the years around one-third of the commissioned peer reviewers have declined to review papers mainly due to increasing professional commitments. We are grateful to the whole international respiratory community for sending us their high quality papers that contributed to the journal's success and please keep sending them! We are leaving a very strong journal in very capable hands with the new Thorax editors Andy Bush and Ian Pavord, to whom we warmly wish every success.

Our objective has been to publish the very best and original respiratory research papers, but we have also strived to provide in Thorax each month something of interest to all those involved with the care of respiratory patients. Back in January 2003, we wrote in our inaugural Thorax Editorial that the success of Thorax will ultimately depend on the influence that the journal has on the readership' and we hope we have achieved the goals and had a positive influence on our readership. We are also privileged that, through the editorship, we have been able to serve our respiratory community both in the UK and abroad. We hope that you agree with us that in September 2010 Thorax can celebrate its position as an important international respiratory journal and that our mission as Thorax Editors has been accomplished.

\section{Competing interests None.}

Provenance and peer review Commissioned; not externally peer reviewed.

Thorax 2010;65:755-756

doi:10.1136/thx.2010.146662

\title{
Diagnosing lung cancer earlier in the UK
}

\section{Richard B Hubbard, David R Baldwin}

The 30326 deaths from lung cancer in England and Wales in 2008 represent 22\% of the total mortality burden from cancer in that year. ${ }^{1}$ The next two most common causes of cancer death were colorectal cancer $(10 \%)$ and breast cancer (8\%). These familiar statistics outline the enormous impact that lung cancer continues to have on public health in the UK.

The dramatic fall in the prevalence of smoking in the UK over the last 60 years means that the lung cancer epidemic in the

Division of Respiratory Medicine, University of Nottingham, Nottingham, UK

Correspondence to Dr Richard Hubbard, Division of Respiratory Medicine, Clinical Sciences Building, City Hospital, Hucknall Road, Nottingham NG5 1PB, UK; richard.hubbard@nottingham.ac.uk
UK has peaked, particularly in men, and this is good news. ${ }^{2}$ In order to protect future generations of people in the UK from developing lung cancer, however, steps to drive down the prevalence of smoking as much as possible, and across all sectors of society, remains a pressing public health priority. ${ }^{34}$ This will inevitably need a broad spectrum of approaches. ${ }^{5} 6$

People who are currently at high risk of developing lung cancer, but do not yet have the disease, may benefit in the future if an effective screening programme can be developed to detect early subclinical disease. At the moment the use of CT imaging seems the approach most likely to succeed, but no trial yet has been able to demonstrate a reduction in mortality. A number of trials are currently ongoing, however, and more definitive evidence is expected between 2012 and 2016. ${ }^{7-13}$ Recently, a feasibility study for a randomised trial of CT screening in the UK has been completed and currently the arrangements are being finalised to undertake a pilot randomised trial. We hope that this project will stimulate more research in this area in the UK, including a trial powered to detect a mortality benefit of screening as well as research into the natural history of early lung cancer detected by CT screening. ${ }^{14} 15$ Research into other screening approaches using more innovative methods is also ongoing but at an early stage. ${ }^{16} 17$

Unfortunately, screening will not help people who are being diagnosed with lung cancer now in the UK. Consequently, those of us who work in healthcare have an important duty of care to these people to ensure that the cancer diagnosis is made at the earliest possible moment and that the most effective currently available treatments are given in a timely fashion in order to improve survival and/or palliate disease. With this in mind, a number of papers published in Thorax have recently 
focused on potential delays in the care of people with lung cancer.

The recent paper by Holmberg et al suggests that people with lung cancer in England do worse than people with lung cancer elsewhere in Europe. ${ }^{18}$ In this study lung cancer registry data from England, Norway and Sweden were analysed and the authors found that, for both men and women and for people in all age groups, the 5-year survival from lung cancer was lower in England than in Norway or Sweden. The median age of diagnosis was also higher in England than in Norway or Sweden. Most of the difference in survival between the countries was the result of particularly poor early survival in England. The researchers conducted a number of sensitivity analyses to allow for the fact that registry data are collected in slightly different ways between the countries, but none of these affected the overall findings greatly. In particular, in England and Norway but not in Sweden, cases that are recorded on the death certificate but not recorded elsewhere are included in the registry dataset. For this reason, all such cases from Norway and England were excluded from the main analyses although, interestingly, the proportion of death certificate-only cases was higher in England (6.8\%) than in Norway (1.0\%). Overall, the authors concluded that clinically relevant differences in survival are present between the countries and that access to healthcare services and/or population awareness are likely to be the main drivers of these differences.

Similar conclusions come from another study published in Thorax which used very different methods. ${ }^{19}$ Imperatori et al compared people presenting with a new diagnosis of lung cancer in 2000 to hospitals in Teesside in the North of England with Varese in Northern Italy. The differences between the two patient groups were striking. People with lung cancer in Teesside were an average of 2 years older than those with lung cancer in Varese and were more likely to have a comorbid illness, to have a worse performance status, a lower forced expiratory volume in $1 \mathrm{~s}$ and to be diagnosed on the basis of symptoms. People in Teesside were also less likely to have histological confirmation of their disease and tended to have a more advanced disease stage at diagnosis. Perhaps not surprisingly, the surgical resection rate and median survival were both higher for people in Varese than for those in Teesside.

These two studies both show that people with lung cancer in the UK present late and do badly, but they do not tell us why. To improve the care for people with lung cancer in the UK, we need to understand the extent to which late presentation is due to population awareness factors or deficiencies in the healthcare system. In other words, is it the public, the health service, or a bit of both? Research to date in this area has been limited and this needs to change because, if we can understand where and why the delays occur in the diagnosis of lung cancer, then there is scope for improving the care and prognosis for people with lung cancer using the currently available treatments.

Information on possible patient-related reasons for delays comes from a study by Corner et al who interviewed 23 patients with recently diagnosed lung cancer and compared their recollection of the emergence of their respiratory symptoms and their interaction with their general practitioner with information from the primary and secondary healthcare records. ${ }^{20}$ The most common symptoms that patients reported were cough $(68 \%)$, breathing changes (68\%) and chest pain (55\%). In total, more than 30 different symptoms were reported and the median time between the self-reported onset of the symptom and diagnosis was 12 months. In contrast, the median time delay between the symptom which triggered the presentation to the general practitioner and the diagnosis was only 2 months. The authors conclude that people with lung cancer often have symptoms for a considerable period of time before they consult their general practitioner, and that this is a major source of delay in the diagnostic process. They recommend that education is needed for smokers to increase the awareness of how lung cancer presents and to seek help. Similar conclusions were drawn by the authors of an interview survey of 360 Scottish people with newly diagnosed lung cancer. In this study Smith et al found that about half the people with lung cancer had symptoms for more than 14 weeks before they presented to their general practitioner. ${ }^{21}$ Those who lived alone, had chronic obstructive pulmonary disease or longer smoking histories tended to have longer times between the onset of symptoms and consulting their general practitioner. There has been very little research directed at the interaction between people with lung cancer and general practitioners in the period up to the time of diagnosis. In one case-control study of 247 people with lung cancer and
1235 age- and sex-matched controls registered with 21 general practices in Exeter, a number of symptoms (haemoptysis, loss of weight, loss of appetite, dyspnoea, chest pain, fatigue, cough), a clinical sign (clubbing) and abnormal investigations (thrombocytosis and abnormal spirometry) all predicted the presence of lung cancer in the 2 years before the cancer was diagnosed. $^{22}$ The researchers then ignored the last 180 days of consultations before the cancer diagnoses to test the ability of these variables to predict lung cancer at an earlier stage, and the factors that remained associated with lung cancer diagnoses were haemoptysis, dyspnoea, abnormal spirometry and being a current or ex-smoker. This in turn raises the possibility that these variables, and perhaps others, could be used to help guide general practitioners as to the risk of lung cancer in the people who consult them, perhaps by developing a predictive scoring system.

Although the currently available evidence is limited, it does suggest that lung cancer could be diagnosed earlier by improving both public awareness of lung cancer and by helping general practitioners to risk stratify the people who consult them for their risk of lung cancer. One example of a public health intervention designed to tackle both of these approaches is the early intervention in lung cancer within Doncaster (ElCiD) project. $^{23}$ This project involved people from local public health departments, secondary care respiratory medicine, nursing, Sheffield Hallam University and a media company. Six areas of Doncaster believed to be at particularly high risk of lung cancer were identified, and a combination of a social marketing campaign to highlight awareness of lung cancer symptoms and training for general practice surgeries around lung cancer was put in place. The provisional results of the project suggest that the campaign led to more people with a troublesome cough visiting their general practitioner and asking for a chest $\mathrm{x}$-ray, more chest x-rays being ordered by general practitioners and an initial increase in the diagnoses of lung cancer. The full results of the study are awaited and will be important in guiding future research and interventions in this area.

In summary, there is an urgent need to understand fully why people in the UK with lung cancer present late and do badly. There is evidence that both patient and health service factors may contribute to these delays. Our aim should be to remove these delays and improve the care 
pathway for people with lung cancer in the UK in order to maximise the number of people who can receive the currently available effective treatments, including potentially curative treatment with surgery or radical radiotherapy. This in turn should then mean that the survival of people with lung cancer in the UK increases to match the best in Europe. This would represent a significant step forward for people with lung cancer in the UK.

Funding RBH's post is funded by the British Lung Foundation via an unrestricted grant from GlaxoSmithKline.

Competing interests None.

Provenance and peer review Commissioned; not externally peer reviewed.

Thorax 2010;65:756-758.

doi:10.1136/thx.2010.145961

\section{REFERENCES}

1. Office for National Statistics. Mortality statistics: deaths registered in 2008. DR 2009.

2. Peto R, Darby S, Deo H, et al. Smoking, smoking cessation, and lung cancer in the UK since 1950: combination of national statistics with two casecontrol studies. BMJ 2000;321:323-9.

3. Raw M, McNeill A, West R. Smoking cessation guidelines for health professionals. A guide to effective smoking cessation interventions for the health care system. Thorax 1998;53(Suppl 5, Pt 1): S1-19.
4. West R, McNeill A, Raw M. Smoking cessation guidelines for health professionals: an update. Thorax 2000;55:987-99.

5. Tobacco Advisory Group of the Royal College of Physicians. Harm reduction in nicotine addition: helping people who can't quit. London: Royal College of Physicians, 2007.

6. World Health Organization. WHO framework convention on tobacco control. Geneva: World Health Organization, 2003.

7. van Klaveren RJ, Oudkerk $M$, Prokop $M$, et al. Management of lung nodules detected by volume CT scanning. N Engl J Med 2009;361:2221-9.

8. Blanchon T, Brechot JM, Grenier PA, et al. Baseline results of the Depiscan study: a French randomized pilot trial of lung cancer screening comparing low dose CT scan (LDCT) and chest X-ray (CXR). Lung Cancer 2007;58:50-8

9. Pedersen JH, Ashraf H, Dirksen A, et al. The Danish randomized lung cancer $\mathrm{CT}$ screening trial: overall design and results of the prevalence round. J Thorac Oncol 2009:4:608-14.

10. Infante M, Cavuto S, Lutman FR, et al. A randomized study of lung cancer screening with spiral computed tomography: three-year results from the DANTE trial. Am J Respir Crit Care Med 2009;180:445-53.

11. Gohagan JK, Marcus PM, Fagerstrom RM, et al. Final results of the Lung Screening Study, a randomized feasibility study of spiral CT versus chest X-ray screening for lung cancer. Lung Cancer 2005;47:9-15

12. Lopes Pegna A, Picozzi G, Mascalchi M, et al. Design, recruitment and baseline results of the ITALUNG trial for lung cancer screening with lowdose CT. Lung Cancer 2009;64:34-40.

13. Infante MV, Pedersen JH. Screening for lung cancer: are we there yet? Curr Opin Pulm Med 2010;16:301-6.
14. Black C, de Verteuil R, Walker S, et al. Population screening for lung cancer using computed tomography, is there evidence of clinical effectiveness? A systematic review of the literature. Thorax 2007;62:131-8

15. Detterbeck $\mathbf{F}$. The fruits of our efforts: time for a different view of lung cancer and CT screening. Thorax 2009;64:465-6.

16. Chapman CJ, Murray A, McElveen JE, et al. Autoantibodies in lung cancer: possibilities for early detection and subsequent cure. Thorax 2008;63:228-33.

17. Mazzone PJ, Hammel J, Dweik R, et al. Diagnosis of lung cancer by the analysis of exhaled breath with a colorimetric sensor array. Thorax 2007;62:565-8

18. Holmberg L, Sandin F, Bray F, et al. National comparisons of lung cancer survival in England, Norway and Sweden 2001-2004: differences occur early in follow-up. Thorax 2010;65:436-41.

19. Imperatori A, Harrison RN, Leitch DN, et al. Lung cancer in Teesside (UK) and Varese (Italy): a comparison of management and survival. Thorax 2006:61:232-9.

20. Corner J, Hopkinson J, Fitzsimmons D, et al. Is late diagnosis of lung cancer inevitable? Interview study of patients' recollections of symptoms before diagnosis. Thorax 2005;60:314-19.

21. Smith SM, Campbell NC, MacLeod U, et al. Factors contributing to the time taken to consult with symptoms of lung cancer: a cross-sectional study. Thorax 2009;64:523-31.

22. Hamilton W, Peters TJ, Round A, et al. What are the clinical features of lung cancer before the diagnosis is made? A population-based case-control study. Thorax 2005;60:1059-65

23. Suckling R. Early lung cancer intervention in Doncaster. Doncaster: Public Health Department, 2008.

\section{Asthma: improved understanding and insights into the challenges of achieving asthma control}

G lan Town, ${ }^{1} \mathrm{~J}$ Mark Fitzgerald ${ }^{2}$

Asthma articles were among the most frequently cited and downloaded items from the Thorax website. The BTS-Sign Asthma Guidelines topped the list, indicating the important role Thorax has played in supporting the respiratory community and the British Thoracic Society in particular.

One of the more important articles covering asthma epidemiology summar-

\footnotetext{
${ }^{1}$ University of Canterbury, Christchurch, New Zealand ${ }^{2}$ UBC Centre for Lung Health, University of British Columbia, Vancouver, Canada
}

Correspondence to J Mark Fitzgerald, UBC Centre for Lung Health, University of British Columbia, Vancouver, Canada; mark.fitzgerald@vch.ca ised the findings of Phase III of the International Study of Asthma and Allergies in Children (ISAAC). ${ }^{1}$ This study, undertaken in some 106 centres in 56 countries, compared the prevalence of asthma symptoms $5-10$ years after the original surveys. In 6- to 7-year olds, asthma prevalence (wheeze in the last 12 months) was $11.6 \%$ and in the 13- to 14-year-old children $13.7 \%$. Comparing trends over time, prevalence had fallen in many western countries but had increased in some other countries including Eastern Europe and parts of Asia. These important ecological data triggered much discussion about the likely causes of the observed trends but failed to generate a unifying hypothesis that could be tested. Thorax also carried a number of other papers generated by the ISAAC study, among the more interesting of which examined the effect of diet on asthma and allergic sensitisation. ${ }^{2}$ This report, based on ISAAC Phase II data, examining $>50000$ children demonstrated that fruit intake was associated with a lower prevalence of current wheeze and conferred a lifetime protective effect, as did consumption of vegetables and fish. We have high expectations that the enormous epidemiological database held by the ISAAC researchers will continue to shed light on risk factors and inform protective strategies.

Asthma genetics was a very hot topic, and interest peaked over the ADAMB3 story. A frenzy of interest ensued and this was touted as a major breakthrough in our understanding. With the benefit of hindsight, the real impact was modest. Breaking initially in Nature in $2002,{ }^{3}$ the gene encoding $\mathrm{ADisintegrin}$ and Metalloprotease 33 , on chromosome $20 \mathrm{p} 13$, was identified by positional cloning. Thorax carried an editorial on the topic the following year, ${ }^{4}$ which noted that this 